\title{
Logic Synthesis of EXOR Projected Sum of Products
}

\author{
Anna Bernasconi ${ }^{1}$, Valentina Ciriani $^{2}$, and Roberto Cordone ${ }^{2}$ \\ 1 Department of Computer Science, University of Pisa Italy, annab@di.unipi.it \\ 2 DTI, University of Milano Italy, \{ciriani, cordone\}@dti.unimi.it
}

\begin{abstract}
We define a new algebraic form for Boolean function representation, called EXOR-Projected Sum of Products (EP-SOP), consisting in a four level network that can be easily implemented in practice. Deriving an optimal EP-SOP from an optimal SOP form is a $N P^{N P}$-hard problem; nevertheless we propose a very efficient approximation algorithm, which returns, in polynomial time, an EP-SOP form whose cost is guaranteed to be near the optimum. Experimental evidence shows that for about $35 \%$ of the classical synthesis benchmarks, EP-SOP networks have a smaller area and delay with respect to the optimal SOPs (sometimes gaining even $40-50 \%$ of the area). Since the computational times required are extremely short, we recommend the use of the proposed approach as a post-processing step after SOP minimization.
\end{abstract}

\section{Introduction}

The classical approach to logic synthesis is the minimization of two-level SOP networks $[2,4,13]$. In this framework the resulting networks have a very low delay, thanks to the fixed number of levels, and the SOP expressions can be computed in a reasonable amount of time. To build networks with a more compact area, multi-level network synthesis has been proposed and widely studied $[8,17]$. The drawbacks of this approach are the unbounded number of levels (and therefore the longer delay), as well as the much larger computational time required to synthesize the network. In an attempt to establish an effective trade-off between these two opposite approaches, recent studies have proposed the optimization of networks with a fixed number of levels (typically, three or four levels) $[1,5-7,12$, 14,16]. Three levels of logic are enough to produce a minimal network for most of the Boolean functions; and in many cases three-level logic is a good compromise between circuit speed, circuit size, and the time needed for the minimization procedure [15]. Three and four-level logic networks are typically more compact than the corresponding SOPs, but the computational time required to compute them can be much longer.

The aim of this paper is to define a network with a bounded number of levels that can be easily implemented in practice and synthesized in a competitive time with respect to two-level synthesis. For this purpose, we propose a four-level network, EXOR-Projected Sum of Products (EP-SOP), which can be built in a very fast post-processing step from an optimal two-level SOP. We first define

Please use the following format when citing this chapter:

Bernasconi, A., Ciriani, V. and Cordone, R., 2007, in IFIP International Federation for Information Processing, Volume 249, VLSI-SoC: Research Trends in VLSI and Systems on Chip, eds. De Micheli, G., Mir, S., Reis, R., (Boston: Springer), pp. 241-257 
the algebraic form of EP-SOP networks, and prove that deriving an optimal EP-SOP from an optimal SOP form is a hard problem $\left(N P^{N P}\right.$-hard). We then describe an approximation algorithm, which returns in polynomial time an EPSOP form whose cost is guaranteed to be near the optimum. Our experimental results show that in about $35 \%$ of the classical synthesis benchmarks the EPSOP obtained has area and delay smaller then those of an optimal SOP form (sometimes gaining even $40-50 \%$ of the area). The computational times required are extremely short, thus recommending the use of this approach as a postprocessing step after SOP minimization.

Before defining EP-SOP forms, we introduce them informally through an example. Let us consider the Boolean function $f$ shown on the left side of Figure 1. An optimal SOP representation for $f$ is $\phi=\bar{x}_{1} x_{2} \bar{x}_{3}+x_{1} \bar{x}_{2} \bar{x}_{3}+\bar{x}_{1} \bar{x}_{2} x_{3}+x_{1} x_{2} x_{3}+$ $x_{3} \bar{x}_{4}$. The right side of Figure 1 represents the projections of $f$ onto the two subspaces where $x_{1}=x_{2}$ and $x_{1} \neq x_{2}$, respectively. As described in the Karnaugh maps on the right side of the figure, the projection of $f$ onto the space $x_{1} \neq x_{2}$ is covered by the optimal SOP form $\bar{x}_{3}+x_{3} \bar{x}_{4}$, while the projection onto the space $x_{1}=x_{2}$ is covered by $x_{3}$. Notice that both SOP forms are much more compact than the original one, because minterms, which were not adjacent in the original Karnaugh map, now merge into new larger prime cubes. For example, the two products $\bar{x}_{1} x_{2} \bar{x}_{3}$ and $x_{1} \bar{x}_{2} \bar{x}_{3}$, which cannot be merged in the original Karnaugh map, correspond to the products $x_{2} \bar{x}_{3}$ and $\bar{x}_{2} \bar{x}_{3}$, which can be unified into product $\bar{x}_{3}$ in the lower Karnaugh map on the right side.

Since the two subspaces, $x_{1}=x_{2}$ and $x_{1} \neq x_{2}$, have characteristic functions equal to $\left(x_{1} \oplus \bar{x}_{2}\right)$ and $\left(x_{1} \oplus x_{2}\right)$, respectively, $f$ can be expressed as $f \equiv\left(x_{1} \oplus \bar{x}_{2}\right) x_{3}+\left(x_{1} \oplus x_{2}\right)\left(\bar{x}_{3}+\bar{x}_{4}\right)$. Figure 2 shows how this form can be easily implemented by using a single 2 -fan in EXOR gate and two PLAs.

As the previous example shows, the products of a generic SOP $\phi$ can be classified into two subsets: those that are entirely included into one of the two subspaces $x_{1}=x_{2}$ and $x_{1} \neq x_{2}$ (for example, in Figure 1 the product $\bar{x}_{1} x_{2} \bar{x}_{3}$ belongs entirely to the subspace $x_{1} \neq x_{2}$ ) and those that intersect both of them, which we will call crossing products (for example, in Figure 1 the product $x_{3} \bar{x}_{4}$ ). In general, it is not always convenient to project a crossing product, since this produces two smaller products, which reside into both subspaces. Therefore, we can choose whether projecting the crossing products or keeping them unprojected. In the second case, the resulting expression also includes a SOP form (called remainder) containing all the crossing products. We call the overall form EP-SOP with remainder.

Figure 3 reports the same example of Figure 1, in which the only crossing product $x_{3} \bar{x}_{4}$ is not projected. In this case, the resulting EP-SOP with remainder form is $f \equiv\left(x_{1} \oplus \bar{x}_{2}\right) x_{3}+\left(x_{1} \oplus x_{2}\right) \bar{x}_{3}+x_{3} \bar{x}_{4}$.

We can observe that EP-SOP expressions can be seen as Boolean factorized forms. Factorization of literal terms is a widely studied field in multi-level logic $[3,17]$. Most of the proposed methods produce disjoint factorization (see [8] for an introduction). In contrast, the factorization of an EP-SOP form is not disjoint since a literal can stay simultaneously in the projected SOPs and in the 


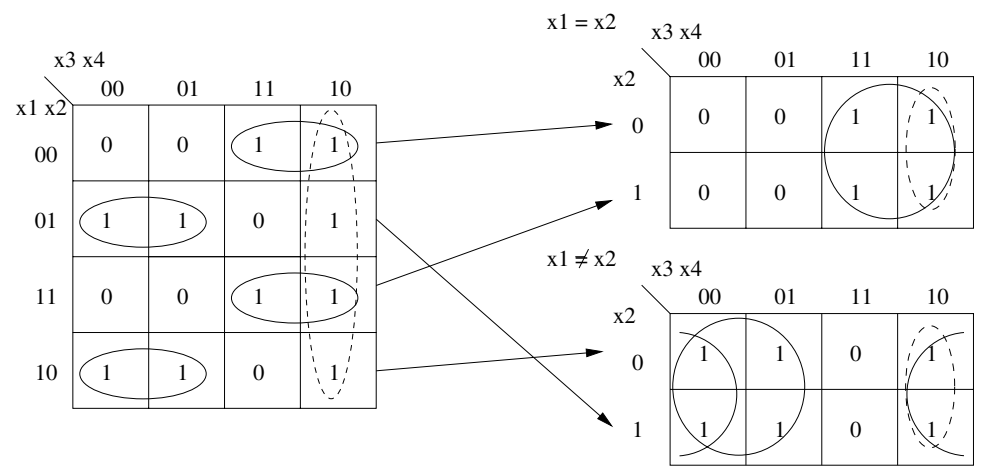

Fig. 1. Karnaugh maps of a function $f$ (left side) and its projections onto $x_{1} \oplus \bar{x}_{2}$ (right side, top) and $x_{1} \oplus x_{2}$ (right side, bottom).

corresponding EXORs. For example, in the EP-SOP form $\left(x_{1} \oplus \bar{x}_{2}\right)\left(x_{2} \bar{x}_{4}+\right.$ $\left.\bar{x}_{3} x_{4}\right)+\left(x_{1} \oplus x_{2}\right)\left(x_{2} \bar{x}_{3}+\bar{x}_{3} \bar{x}_{4}\right)$ the literal $x_{2}$ appears both in the EXORs and in the SOPs.

Finally, EP-SOP forms share some similarities with another model of Boolean function representation: the Linearly-Transformed BDDs [10,11]. LTBDDs are binary decision diagrams whose nodes are labeled with EXORs of variables, instead of just single variables. Thus, the node on the first level of a LTBDD, if labeled with an EXOR, defines the same kind of decomposition on which EP-SOPs are based.

The remainder of this paper is organized as follows. Section 2 describes the algebraic expression for EP-SOPs with and without remainder. Section 3 characterizes the computational complexity of the problem. Section 4 presents an approximation algorithm for EP-SOP synthesis, and proves that its solution is nearly optimal. In the end, Section 5 discusses the experimental results.

\section{EP-SOP representation of Boolean functions}

The following two sections formally describe EP-SOP expressions with and without remainder, and show how to derive them from an original optimal SOP form.

\section{$2.1 \quad$ EP-SOP without remainder}

Let us consider a SOP form $\phi$, and a couple of variables $x_{i}$ and $x_{j}$, where without loss of generality $i<j$. The space $\{0,1\}^{n}$ can be partitioned into two disjoint subspaces: the space defined by the characteristic function $\chi_{\bar{\oplus}}=\left(x_{i} \oplus \bar{x}_{j}\right)$, i.e., the space where $x_{i}=x_{j}$, and its complement defined by the function $\chi_{\oplus}=$ $\left(x_{i} \oplus x_{j}\right)$, i.e., the space where $x_{i} \neq x_{j}$.

We can write $\phi$ as the sum (union) of its two projections, $\phi_{\oplus}$ and $\phi_{\bar{\oplus}}$, onto these two spaces. Even if the projections allow us to eliminate a variable ad 


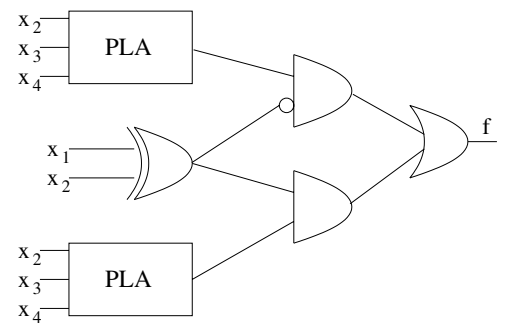

Fig. 2. EP-SOP-network without remainder for the function in Figure 1.

libitum between $x_{i}$ and $x_{j}$, we always remove $x_{i}$ (the one with lower index). In order to perform the two projections we must project one by one the products $p \in \phi$, considering four cases.

Algorithm 1 (Project. onto $\left(x_{i} \oplus \bar{x}_{j}\right)$ and $\left(x_{i} \oplus x_{j}\right)$ for EP-SOP) Given a $S O P$ form $\phi=p_{1}+p_{2}+\cdots p_{m}$, for each $p$ in $\left\{p_{1}, p_{2}, \ldots, p_{m}\right\}$ project $p$ in $\phi_{\oplus}$ or in $\phi_{\bar{\oplus}}$ using the following strategy:

1. If $p$ contains both $x_{i}$ and $x_{j}$ (possibly complemented), i.e., $p=x_{i} x_{j} q$, where $q$ is a product of literals, $p$ has no projection onto the subspace where $x_{i} \neq x_{j}$. Thus, no product will be added to $\phi_{\oplus}$. By contrast, the projection of $p=x_{i} x_{j} q$ onto the subspace where $x_{i}=x_{j}$ gives the product $x_{j} q$, which will be added to $\phi_{\bar{\oplus}}$. The three other cases $\left(p=x_{i} \bar{x}_{j} q, p=\bar{x}_{i} x_{j} q, p=\bar{x}_{i} \bar{x}_{j} q\right)$ can be handled in a similar way.

2. If $p$ contains $x_{i}$ (possibly complemented) and not $x_{j}$, i.e., $p=x_{i} q$, where $q$ is a product of literals, the projection of $p$ onto the subspace where $x_{i} \neq x_{j}$ gives the product $\bar{x}_{j} q$, which will be added to $\phi_{\oplus}$. The projection of $p$ onto the subspace where $x_{i}=x_{j}$ gives the product $x_{j} q$, which will be added to $\phi_{\bar{\oplus}}$. The other case $\left(p=\bar{x}_{i} q\right)$ can be handled in a similar way: $x_{j} q$ will be added to $\phi_{\oplus}$, and $\bar{x}_{j} q$ will be added to $\phi_{\bar{\oplus}}$.

3. If $p$ contains $x_{j}$ (possibly complemented) and not $x_{i}$, i.e., $p=x_{j} q$, where $q$ is a product of literals, the projections of $p$ onto both subspaces leave the product unchanged, thus $p=x_{j} q$ will be added to both $\phi_{\oplus}$ and $\phi_{\bar{\oplus}}$. The other case $\left(p=\bar{x}_{j} q\right)$ can be handled in the same way, by adding $p$ to both $\phi_{\oplus}$ and $\phi \bar{\oplus}$.

4. If $p$ does not contain $x_{i}, \bar{x}_{i}, x_{j}, \bar{x}_{j}$, the projections of $p$ onto both subspaces leave the product unchanged, thus $p$ will be added to both $\phi_{\oplus}$ and $\phi_{\bar{\oplus}}$.

Observe that the last three type of products are indeed crossing products, which are projected onto the two spaces, while the products containing both $x_{i}$ and $x_{j}$ are projected only onto one of them.

Example 1. Let us consider the Boolean function $f$ shown on the left side of Figure 1. An optimal SOP representation for $f$ is $\phi=\bar{x}_{1} x_{2} \bar{x}_{3}+x_{1} \bar{x}_{2} \bar{x}_{3}+$ $\bar{x}_{1} \bar{x}_{2} x_{3}+x_{1} x_{2} x_{3}+x_{3} \bar{x}_{4}$. Suppose to project $\phi$ onto the spaces $\left(x_{1} \oplus \bar{x}_{2}\right)$ and 
$\left(x_{1} \oplus x_{2}\right)$. The first product in $\phi$ contains both $x_{1}$ and $x_{2}$, thus it is not a crossing product (strategy 1 of Algorithm 1). Since $x_{1}$ is complemented and $x_{2}$ is not complemented we project this product onto the space $\left(x_{1} \oplus x_{2}\right)$ (in fact, $\left.x_{1} \neq x_{2}\right)$. The projected product is $x_{2} \bar{x}_{3}$. The unique crossing product of $\phi$ is $x_{3} \bar{x}_{4}$, since it does not contain $x_{1}$ and $x_{2}$. This product will be inserted in both the spaces without removing any literal. The overall projection will return the form $\left(x_{1} \oplus \bar{x}_{2}\right)\left(\bar{x}_{2} x_{3}+x_{2} x_{3}+x_{3} \bar{x}_{4}\right)+\left(x_{1} \oplus x_{2}\right)\left(x_{2} \bar{x}_{3}+\bar{x}_{2} \bar{x}_{3}+x_{3} \bar{x}_{4}\right)$. Note that the SOP forms of the projected spaces are not minimal. Minimizing them we obtain $\left(x_{1} \oplus \bar{x}_{2}\right) x_{3}+\left(x_{1} \oplus x_{2}\right)\left(\bar{x}_{3}+x_{3} \bar{x}_{4}\right)$.

We can now formally define the EP-SOP expressions. These forms can be derived starting from a SOP representation $\phi$ of a Boolean function $f$ in two steps.

First we project $\phi$ onto the two subspaces $\left(x_{i} \oplus x_{j}\right)$ and $\left(x_{i} \oplus \bar{x}_{j}\right)$, as explained before, and we obtain the following expression.

Definition 1. Let $f:\{0,1\}^{n} \rightarrow\{0,1\}$, and let $\phi$ be a SOP representation of $f$. Given a couple of variables $x_{i}$ and $x_{j}$, the $(i, j)$-EP-SOP of $f$ is the expression

$$
\xi_{i j}=\left(x_{i} \oplus x_{j}\right) \phi_{\oplus}+\left(x_{i} \oplus \bar{x}_{j}\right) \phi_{\bar{\oplus}},
$$

where $\phi_{\oplus}$ and $\phi_{\bar{\oplus}}$ are the projections of $\phi$ onto the spaces $\left(x_{i} \oplus x_{j}\right)$ and $\left(x_{i} \oplus \bar{x}_{j}\right)$, respectively.

After the projection we can further minimize the two SOPs $\phi_{\oplus}$ and $\phi_{\bar{\oplus}}$ in order to minimize the EP-SOP $\xi_{i j}$.

Definition 2. Let $f:\{0,1\}^{n} \rightarrow\{0,1\}$, and let $\phi$ be a SOP representation of $f$. Given a couple of variables $x_{i}$ and $x_{j}$, the minimal $(i, j)$-EP-SOP of $f$ is the expression

$$
\xi_{i j}^{(m i n)}=\left(x_{i} \oplus x_{j}\right) \phi_{\oplus}^{(\min )}+\left(x_{i} \oplus \bar{x}_{j}\right) \phi_{\bar{\oplus}}^{(\min )},
$$

where $\phi_{\oplus}^{(\text {min })}$ and $\phi_{\bar{\oplus}}^{(\text {min })}$ are two minimal SOP forms representing the projections of $\phi$ onto the spaces $\left(x_{i} \oplus x_{j}\right)$ and $\left(x_{i} \oplus \bar{x}_{j}\right)$, respectively.

In the previous definitions we have fixed a single couple of variables, but we are interested in finding the minimal EP-SOP representation of a Boolean function, i.e., the expression containing the minimum number of products among all possible minimal EP-SOP with respect to any couple of variables.

Let $|\phi|$ denote the number of products in a $\operatorname{SOP} \phi$, and $|\xi|=\left|\phi_{\oplus}\right|+\left|\phi_{\bar{\oplus}}\right|$ the overall number of products in an EP-SOP $\xi$.

Definition 3. The minimal EP-SOP representation of a Boolean function $f$ is given by the EP-SOP expression $\xi_{M I N}$ such that

$$
\left|\xi_{M I N}\right|=\min _{i, j}\left|\xi_{i j}^{(m i n)}\right| .
$$




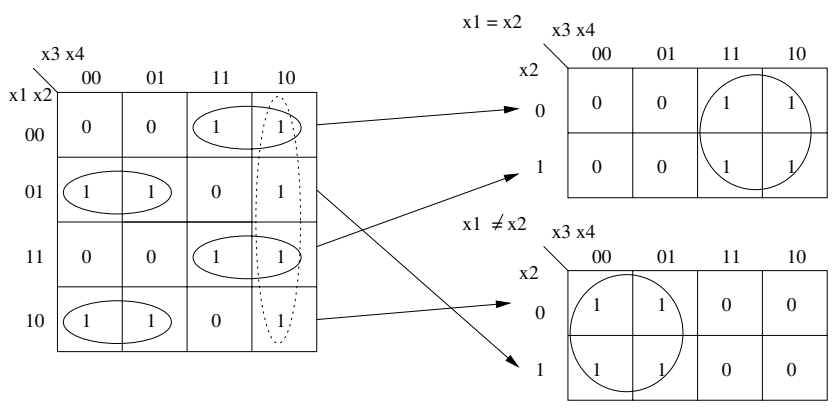

\begin{tabular}{|c|c|c|c|c|}
\hline \multicolumn{5}{|c|}{ Remainder ${ }_{\mathrm{x} 3}$} \\
\hline & 00 & 01 & 11 & 10 \\
\hline 00 & 0 & 0 & 0 & 1 \\
\hline 01 & 0 & 0 & 0 & 1 \\
\hline 11 & 0 & 0 & 0 & 1 \\
\hline 10 & 0 & 0 & 0 & 1 \\
\hline
\end{tabular}

Fig. 3. Karnaugh maps of a function $f$ (left side), its projections onto $x_{1} \oplus \bar{x}_{2}$ (center, top) and $x_{1} \oplus x_{2}$ (center, bottom), and the remainder (right side).

\section{$2.2 \quad$ EP-SOP with remainder}

As already noted, when we project a SOP form onto the two spaces $\left(x_{i} \oplus x_{j}\right)$ and $\left(x_{i} \oplus \bar{x}_{j}\right)$, some products will appear only once in the final expression, precisely the products containing the two literals defining the projection spaces, while the other products (crossing products) will appear twice, one in each projected SOP.

In order to keep the number of products as small as possible, we introduce the notion of EP-SOP with remainder.

Algorithm 2 (Proj. onto $\left(x_{i} \oplus \bar{x}_{j}\right)$ and $\left(x_{i} \oplus x_{j}\right)$ for EP-SOP with rem.) Given a SOP form $\phi=p_{1}+p_{2}+\cdots p_{m}$, for each $p$ in $\left\{p_{1}, p_{2}, \ldots, p_{m}\right\}$ project $p$ in $\phi_{\oplus}$ or in $\phi_{\bar{\oplus}}$, or insert it in the remainder $\rho$ using the following strategy:

1. If $p$ contains both $x_{i}$ and $x_{j}$ (possibly complemented), i.e., $p=x_{i} x_{j} q$, where $q$ is a product of literals, $p$ has no projection onto the subspace where $x_{i} \neq x_{j}$. Thus, no product will be added to $\phi_{\oplus}$. By contrast, the projection of $p=x_{i} x_{j} q$ onto the subspace where $x_{i}=x_{j}$ gives the product $x_{j} q$, which will be added to $\phi_{\bar{\oplus}}$. The three other cases $\left(p=x_{i} \bar{x}_{j} q, p=\bar{x}_{i} x_{j} q, p=\bar{x}_{i} \bar{x}_{j} q\right)$ can be handled in a similar way.

2. Otherwise ( $p$ is a crossing product) insert $p$ in the remainder.

For example, let us consider the Boolean function $f$ shown on the left side of Figure 3. The unique crossing product of $\phi$ is $x_{3} \bar{x}_{4}$ since it does not contain $x_{1}$ and $x_{2}$. This product will be inserted now in the remainder. The overall projection will return the form: $\left(x_{1} \oplus \bar{x}_{2}\right)\left(\bar{x}_{2} x_{3}+x_{2} x_{3}\right)+\left(x_{1} \oplus x_{2}\right)\left(x_{2} \bar{x}_{3}+\bar{x}_{2} \bar{x}_{3}\right)+$ $x_{3} \bar{x}_{4}$. Minimizing the projected SOPs we obtain $\left(x_{1} \oplus \bar{x}_{2}\right) x_{3}+\left(x_{1} \oplus x_{2}\right) \bar{x}_{3}+$ $x_{3} \bar{x}_{4}$.

Formally we have:

Definition 4. Let $f:\{0,1\}^{n} \rightarrow\{0,1\}$, and let $\phi$ be a SOP representation of $f$. Given a couple of variable $x_{i}$ and $x_{j}$, the $(i, j)$-EP-SOP with remainder of $f$ is the expression

$$
\psi_{i j}=\left(x_{i} \oplus x_{j}\right) \phi_{\oplus}^{\prime}+\left(x_{i} \oplus \bar{x}_{j}\right) \phi_{\oplus}^{\prime}+\rho,
$$


where $\phi_{\oplus}^{\prime}$ and $\phi_{\bar{\oplus}}^{\prime}$ are the two projections of the products of $\phi$ containing both $x_{i}$ and $x_{j}$ (possibly complemented) onto the spaces $\left(x_{i} \oplus x_{j}\right)$ and $\left(x_{i} \oplus \bar{x}_{j}\right)$, respectively, and $\rho$ is the sum of all crossing products of $\phi$.

In other words we project onto the subspaces $\left(x_{i} \oplus x_{j}\right)$ and $\left(x_{i} \oplus \bar{x}_{j}\right)$ only the products that entirely reside in them, while the crossing products are not projected, but are inserted in the remainder $\rho$. Again for this form, we can further minimize the projected SOPs $\phi_{\oplus}^{\prime}$ and $\phi_{\bar{\oplus}}^{\prime}$ in order to obtain a more compact expression:

Definition 5. Let $f:\{0,1\}^{n} \rightarrow\{0,1\}$, and let $\phi$ be a SOP representation of $f$. Given a couple of variable $x_{i}$ and $x_{j}$, the minimal $(i, j)$-EP-SOP with remainder of $f$ is the expression

$$
\psi_{i j}^{(m i n)}=\left(x_{i} \oplus x_{j}\right) \phi_{\oplus}^{\prime(m i n)}+\left(x_{i} \oplus \bar{x}_{j}\right) \phi_{\frac{\oplus}{\oplus}}^{(\min )}+\rho^{(m i n)},
$$

where $\phi_{\oplus}^{\prime(\min )}$ and $\phi_{\bar{\oplus}}^{\prime(\min )}$ are two minimal SOP forms representing the projections of the products of $\phi$ containing both $x_{i}$ and $x_{j}$ (possibly complemented) onto the spaces $\left(x_{i} \oplus x_{j}\right)$ and $\left(x_{i} \oplus \bar{x}_{j}\right)$, respectively, and $\rho^{(m i n)}$ is the optimal sum of all other products of $\phi$.

The overall minimal form (with respect to any possible couple of variables) is described as follows. Let $|\psi|$ denote the number of products in an EP-SOP with remainder, i.e., $|\psi|=\left|\phi_{\oplus}^{\prime}\right|+\left|\phi_{\bar{\oplus}}^{\prime}\right|+|\rho|$.

Definition 6. The minimal EP-SOP with remainder representation of a Boolean function $f$ is given by the EP-SOP expression $\psi_{M I N}$ such that

$$
\left|\psi_{M I N}\right|=\min _{i, j}\left|\psi_{i j}^{(\min )}\right| \text {. }
$$

Note that if we start from a minimal SOP, the remainder is already minimal, i.e., the number of its products cannot be further reduced: $\left|\rho^{(\mathrm{min})}\right|=|\rho|$.

We cannot decide in advance which one of the two EP-SOP expressions (with or without remainder) is the more compact. On one hand, if we project the crossing products in the two spaces we could further minimize them. On the other hand it could be more convenient kipping them in the remainder.

For example, consider the minimal SOP form $\phi=\bar{x}_{1} x_{2} \bar{x}_{3}+x_{1} \bar{x}_{2} \bar{x}_{3}+x_{3} \bar{x}_{4}$ and the couple $x_{1}$ and $x_{2}$. The minimal (1,2)-EP-SOP without remainder is $\left(x_{1} \oplus \bar{x}_{2}\right) x_{3} \bar{x}_{4}+\left(x_{1} \oplus x_{2}\right)\left(\bar{x}_{3}+x_{3} \bar{x}_{4}\right)$, while the minimal $(1,2)$-EP-SOP with remainder is $\left(x_{1} \oplus x_{2}\right) \bar{x}_{3}+x_{3} \bar{x}_{4}$. In this case the form with remainder is clearly more compact.

Alternatively consider the minimal SOP form $\phi=\bar{x}_{1} \bar{x}_{2} x_{3} \bar{x}_{4}+x_{1} x_{2} x_{3} \bar{x}_{4}+$ $\bar{x}_{1} x_{2} \bar{x}_{3}+x_{1} \bar{x}_{2} \bar{x}_{3} \bar{x}_{4}+\bar{x}_{1} \bar{x}_{3} x_{4}+x_{2} \bar{x}_{3} x_{4}$ and the couple $x_{1}$ and $x_{2}$. The minimal $(1,2)$-EP-SOP without remainder is $\left(x_{1} \oplus \bar{x}_{2}\right)\left(x_{3} \bar{x}_{4}+\bar{x}_{3} x_{4}\right)+\left(x_{1} \oplus x_{2}\right)\left(x_{2} \bar{x}_{3}+\right.$ $\left.\bar{x}_{3} \bar{x}_{4}\right)$, while the minimal $(1,2)$-EP-SOP with remainder is $\left(x_{1} \oplus \bar{x}_{2}\right)\left(x_{3} \bar{x}_{4}\right)+$ $\left(x_{1} \oplus x_{2}\right)\left(x_{2} \bar{x}_{3}+\bar{x}_{3} \bar{x}_{4}\right)+\bar{x}_{1} \bar{x}_{3} x_{4}+x_{2} \bar{x}_{3} x_{4}$. In this case the form without remainder is more convenient. 


\section{Computational Complexity}

In this section we analyze the computational complexity of the following problem: given a minimal SOP form $\phi$ for a Boolean function $f$ and a couple of variables $x_{i}$ and $x_{j}$, find a minimal $(i, j)$-EP-SOP of $f$.

Since projecting $\phi$ is easy (polynomial), as shown in Section 2, the core of the problem is the minimization of the two projected SOPs. In general $\phi_{\oplus}^{(\min )}$ and $\phi_{\bar{\oplus}}^{(\min )}$ are different from the projections $\phi_{\oplus}$ and $\phi_{\bar{\oplus}}$, even if $\phi$ is already in minimal form. Indeed, projecting the single products of $\phi$, we have no guarantee that the resulting SOP forms $\phi_{\oplus}$ and $\phi_{\bar{\oplus}}$ are still prime and irredundant.

Notice that the more common projections of a minimal SOP form $\phi$ onto the spaces $x_{i}$ and $\bar{x}_{i}$ (Shannon projections) are guaranteed to be minimal. For instance, the projection (Shannon decomposition) with respect to $x_{1}$ and $\bar{x}_{1}$ of the minimal SOP form $\phi=\bar{x}_{1} x_{2} \bar{x}_{3}+x_{1} \bar{x}_{2} \bar{x}_{3}+x_{1} x_{3} \bar{x}_{4}$ is $\bar{x}_{1}\left(x_{2} \bar{x}_{3}\right)+x_{1}\left(\bar{x}_{2} \bar{x}_{3}+\right.$ $\left.x_{3} \bar{x}_{4}\right)$, and the two projected SOP forms are already minimal.

In [19], the decision version of the problem of finding a minimal SOP representation of a Boolean function $f$ starting from any SOP for $f$ (SOP-2-MIN $S O P$ ) has been proved to be $N P^{N P}$-complete. Finding $\phi_{\oplus}^{(\min )}$ and $\phi_{\frac{(m i n)}{\oplus}}^{(\text {from }}$ $\phi_{\oplus}$ and $\phi_{\bar{\oplus}}$ when the starting SOP $\phi$ is minimal, could nevertheless be an easy (polynomial) problem? We show here that the answer to this question is negative, since the problem under study turns out to be at least as difficult as SOP-2-MIN $S O P$. Let us first formally define the two problems.

Problem 1 (SOP-2-MIN SOP).

INSTANCE: A SOP formula $\phi$ and an integer $k$.

QUESTION: Is there a SOP $\phi^{\prime}$ with at most $k$ products and for which $\phi^{\prime} \equiv \phi$ ?

Problem 2 (MIN SOP-2-MIN $(i, j)$-EP-SOP).

INSTANCE: Minimal SOP formula $\phi$, a couple of variables $x_{i}$ and $x_{j}$.

QUESTION: Find the minimal $(i, j)$-EP-SOP $\xi_{i j}^{(\min )}$ :

$$
\xi_{i j}^{(m i n)}=\left(x_{i} \oplus x_{j}\right) \phi_{\oplus}^{(m i n)}+\left(x_{i} \oplus \bar{x}_{j}\right) \phi_{\bar{\oplus}}^{(m i n)} .
$$

The proof of the hardness of MIN SOP-2-MIN $(i, j)-E P-S O P$ is based on the concept of polynomial time Turing reduction, defined as follows.

Definition 7. A problem $\Pi$ is Turing-reducible to a problem $\Pi^{\prime}, \Pi \preceq_{T} \Pi^{\prime}$, if there is an algorithm $A$ that solves $\Pi$ by using a hypothetical subroutine $S$ for solving $\Pi^{\prime}$ such that, if $S$ were a polynomial time algorithm for $\Pi^{\prime}$, then $A$ would be a polynomial time algorithm for $\Pi$.

Let us consider the complexity of the following problem:

Problem 3 ( $M I N S O P+P R O D U C T-2-M I N S O P)$.

INSTANCE: A minimal SOP formula $\phi$ for a Boolean function $f$ and a product $p$. QUESTION: Find a minimal SOP formula for the function $f+p$ 
Lemma 1. MIN SOP+PRODUCT-2-MIN SOP is a NP $P^{N}$-hard problem.

Proof. We show that the problem SOP-2-MIN SOP is Turing-reducible to $M I N$ $S O P+P R O D U C T-2-M I N$ SOP.

Consider a $\operatorname{SOP} \phi=p_{1}+p_{2}+\ldots+p_{m}$ for a function $f$ depending on $n$ variables. In order to find a minimal SOP $\phi^{\prime}$ for $f$, we can proceed iteratively as follows.

First we compute a minimal SOP for the function $p_{1}+p_{2}$. Note that this corresponds to deriving a minimal SOP $\phi^{(1)}$ for the union of the minimal SOP $p_{1}$ and a product $p_{2}$.

In general, step $i$ of this iterative process would consist in computing a minimal SOP $\phi^{(i)}$ for the function $\phi^{(i-1)}+p_{i}$, defined as the union of a minimal SOP, $\phi^{(i-1)}$, and the product $p_{i}$.

If we could perform this step in polynomial time, then we could solve the problem $S O P-2-M I N S O P$ in polynomial time, performing $m-1$ iterations.

Based on the previous lemma, we can now prove our main complexity result.

Theorem 1. MIN SOP-2-MIN $(i, j)$-EP-SOP is NP $P^{N P}$-hard.

Proof. To prove the thesis, it is enough to show that the MIN SOP+PRODUCT2-MIN SOP problem is Turing-reducible to MIN SOP-2-MIN $(i, j)$-EP-SOP.

Consider a minimal SOP $\phi^{(\min )}$ for a function $f$, depending on $n$ variables $x_{1}, x_{2}, \ldots, x_{n}$, and a product $p$. Then consider the SOP

$$
\Phi=x_{n+1} \phi^{(\min )}+x_{n+2} p,
$$

where $x_{n+1}$ and $x_{n+2}$ are two additional variables. Suppose that $\phi^{(\min )}$ contains $t$ products.

First of all observe that $\Phi$ is a minimal SOP form. Indeed, $x_{n+1} \phi^{(\min )}$ is minimal and does not cover the points of the cube described by $\bar{x}_{n+1} x_{n+2} p$. Thus we need at least a product to cover these points. This means that a minimal SOP must contain at least $t+1$ products, and this immediately implies that $\Phi$ is minimal.

Now, let us derive an EP-SOP from $\Phi$ with respect to the couple of additional variables $x_{n+1}$ and $x_{n+2}$. We get the following expression:

$\xi_{n+1, n+2}=\left(x_{n+1} \oplus x_{n+2}\right)\left(\bar{x}_{n+2} \phi^{(m i n)}+x_{n+2} p\right)+\left(x_{n+1} \oplus \bar{x}_{n+2}\right)\left(x_{n+2} \phi^{(m i n)}+x_{n+2} p\right)$.

If we could derive $\xi_{n+1, n+2}^{(\min )}$ in polynomial time, then we would be able to minimize the two expressions $\left(\bar{x}_{n+2} \phi^{(\min )}+x_{n+2} p\right)$ and $\left(x_{n+2} \phi^{(\min )}+x_{n+2} p\right)$ in polynomial time. This implies that we could solve in polynomial time an instance of MIN SOP +PRODUCT-2-MIN SOP since, from the second expression, we have $\left(x_{n+2} \phi^{(\min )}+x_{n+2} p\right)^{(\min )}=x_{n+2} \cdot\left(\phi^{\text {min }}+p\right)^{(\min )}$. 


\section{Polynomial time approximation algorithms}

In the previous section we have shown that, even if we start from a minimal SOP form and we fix a couple of variables $x_{i}$ and $x_{j}$, finding a minimal $(i, j)$-EP-SOP is a hard problem. In this section we will show how it is possible to find a good solution to the latter problem in polynomial time.

In a minimization framework, a $p$-approximation algorithm (i.e., an algorithm with approximation ratio $p$ ) guarantees that the $\operatorname{cost} C$ of its solution is such that $C / C^{*} \leq p$, where $C^{*}$ is the cost of an optimal solution [9]. Both heuristics and approximation algorithms do not guarantee the minimality of their solution, but while we cannot perform any evaluation on the result of a heuristic, an approximation algorithm gives guaranteed near-optimum solutions.

We now describe a polynomial approximation algorithm for the problem of finding the minimal EP-SOP (minimal EP-SOP with remainder) representation of a function $f$ starting from a minimal $S O P \phi$ for $f$ that guarantees an approximation ratio of $4(2)$. The main idea is to select the most frequent couple of variables in the minimal SOP representation, and project the expression with respect to this couple. The two projected SOPs will be further synthesized with a SOP polynomial heuristic. The overall algorithm is described below.

Algorithm 3 (Approximation Algorithm) Given a minimal SOP expression $\phi$ :

Step 1 Select the couple of variables $x_{i}$ and $x_{j}$ simultaneously appearing (possibly complemented) with the highest frequency in the products of $\phi$.

Step 2 Project $\phi$ onto the spaces $\left(x_{i} \oplus x_{j}\right)$ and $\left(x_{i} \oplus \bar{x}_{j}\right)$ as described in Algorithms 1 or 2.

Step 3 Minimize the two projected SOPs using a polynomial time heuristic (e.g., Espresso NOT EXACT).

Notice that the two versions (with and without remainder) differ only in the projection Step 2 discussed in Section 2. The three steps can be performed in polynomial time.

We now prove that the proposed synthesis strategy is indeed an approximation algorithm for the two EP-SOP minimization problems.

Consider first the problem without remainder. In order to prove that the $\operatorname{cost}\left|\xi_{i j}^{(m i n)}\right|$ of our solution is such that $\left|\xi_{i j}^{(m i n)}\right| /\left|\xi_{M I N}\right|$ is upper bounded by a constant, where $\left|\xi_{M I N}\right|$ is the cost of an optimal solution, we first find a lower bound for $\left|\xi_{M I N}\right|$, as shown in Lemma 2 and then an upper bound for $\left|\xi_{i j}^{(m i n)}\right|$, as shown in Theorem 2. We follow a similar strategy for EP-SOPs with remainder using Lemma 3 and Theorem 3.

Let us consider a minimal SOP form $\phi$ for a Boolean function $f$ and a minimal EP-SOP without remainder $\xi_{M I N}$.

Lemma 2.

$$
\left|\xi_{M I N}\right| \geq \frac{1}{2}|\phi|
$$


Proof. Let us suppose that the variables $x_{h}$ and $x_{k}$ are such that

$$
\xi_{h k}^{(m i n)}=\left(x_{h} \oplus x_{k}\right) \phi_{\oplus}^{(\min )}+\left(x_{h} \oplus \bar{x}_{k}\right) \phi_{\frac{(\min )}{\oplus}}=\xi_{M I N} .
$$

We build a SOP $\phi_{h k}$ starting from $\xi_{h k}^{(\min )}$. Let $\phi_{\oplus}^{(m i n)}=\sum_{i=1}^{\left|\phi_{\oplus}^{(m i n)}\right|} p_{i}$ and $\phi_{\oplus}^{(m i n)}=$ $\sum_{i=1}^{\left|\phi_{\bar{\oplus}}^{(m i n)}\right|} q_{i}$. Thus

$$
\begin{aligned}
\phi_{h k} & =\left(x_{h} \oplus x_{k}\right) \sum_{i=1}^{\left|\phi_{\oplus}^{(m i n)}\right|} p_{i}+\left(x_{h} \oplus \bar{x}_{k}\right) \sum_{i=1}^{\left|\phi_{\bar{\oplus}}^{(m i n)}\right|} q_{i} \\
& =x_{h} \bar{x}_{k} \sum_{i=1}^{\left|\phi_{\oplus}^{(m i n)}\right|} p_{i}+\bar{x}_{h} x_{k} \sum_{i=1}^{\left|\phi_{\oplus}^{(m i n)}\right|} p_{i}+x_{h} x_{k} \sum_{i=1}^{\left|\phi_{\bar{\oplus}}^{(m i n)}\right|} q_{i}+\bar{x}_{h} \bar{x}_{k} \sum_{i=1}^{\left|\phi_{\bar{\oplus}}^{(m i n) \mid}\right|} q_{i} .
\end{aligned}
$$

Since $\phi$ is minimal, we have that $\left|\phi_{h k}\right| \geq|\phi|$. Moreover, since

$$
\left|\phi_{h k}\right|=2\left|\xi_{h k}^{(\min )}\right|=2\left(\left|\phi_{\oplus}^{(m i n)}\right|+\left|\phi_{\frac{(m i n)}{\oplus}}^{(m)} \geq\right| \phi \mid,\right.
$$

the thesis immediately follows:

$$
\left|\xi_{M I N}\right|=\left|\xi_{h k}^{(\min )}\right|=\left|\phi_{\oplus}^{(\min )}\right|+\left|\phi_{\bar{\oplus}}^{(\min )}\right| \geq \frac{1}{2}|\phi| .
$$

A similar result holds for the EP-SOPs with remainder.

\section{Lemma 3.}

$$
\left|\psi_{M I N}\right| \geq \frac{1}{2}(|\phi|+|\rho|),
$$

where $\rho$ is the remainder of $\psi_{M I N}$.

Proof. Let us suppose that the EP-SOP form

$$
\psi_{h k}^{(\min )}=\left(x_{h} \oplus x_{k}\right) \phi_{\oplus}^{\prime(\min )}+\left(x_{h} \oplus \bar{x}_{k}\right) \phi_{\bar{\oplus}}^{\prime(\min )}+\rho
$$

is minimal, with respect to the overall number of products, among all other EP-SOPs with remainder, i.e., $\psi_{h k}^{(\min )}=\psi_{M I N}$.

As in the proof of Lemma 2, we derive a SOP representation $\phi_{h k}$ for $f$ from $\psi_{h k}^{(\min )}$, and we get

$$
\left|\phi_{h k}\right|=2\left(\left|\phi_{\oplus}^{\prime(\min )}\right|+\mid \phi_{\frac{\prime(m i n)}{\oplus}}^{\prime(\min }\right)+|\rho| \geq|\phi| .
$$

Thus

$$
\left|\phi_{\oplus}^{\prime(\min )}\right|+\left|\phi_{\frac{\prime(\min )}{\oplus}}\right|+\frac{1}{2}|\rho| \geq \frac{1}{2}|\phi|,
$$

and we immediately derive

$$
\left|\psi_{M I N}\right|=\left|\psi_{h k}^{(\min )}\right|=\left|\phi_{\oplus}^{\prime(m i n)}\right|+\left|\phi_{\bar{\oplus}}^{\prime(\min )}\right|+|\rho| \geq \frac{1}{2}(|\phi|+|\rho|) .
$$


We now prove that if we project the starting minimal SOP $\phi$ with respect to the couple of variables $x_{i}$ and $x_{j}$ simultaneously appearing (possibly complemented) with the highest frequency in its products, we get a solution whose approximation ratio in the worst case is bounded by 4 for the EP-SOP without remainder, and by 2 for the EP-SOP with remainder.

For a couple of variables $x_{i}$ and $x_{j}$, let us denote with $\nu_{i j}$ the number of products in $\phi$ containing both $x_{i}$ and $x_{j}$, possibly complemented.

Theorem 2. Let $\xi_{M I N}$ be a minimal EP-SOP of a Boolean function $f$, and $\phi$ a minimal SOP form for $f$. Let $\xi_{i j}^{(\min )}$ be the minimal $(i, j)$-EP-SOP derived with respect to the couple of variables $\left(x_{i}\right.$ and $\left.x_{j}\right)$ appearing with the highest frequency in the products of $\phi$. Then

$$
\frac{\left|\xi_{i j}^{(\min )}\right|}{\left|\xi_{M I N}\right|} \leq \frac{\left|\xi_{i j}\right|}{\left|\xi_{M I N}\right|} \leq 4-\frac{2 \nu_{i j}}{|\phi|} .
$$

Proof. Observe that

$$
\left|\xi_{i j}^{(\min )}\right| \leq\left|\xi_{i j}\right| \leq 2|\phi|-\nu_{i j},
$$

since the $\nu_{i j}$ products containing the two variables $x_{i}$ and $x_{j}$ appear only once in $\xi_{i j}$, while all other products appear twice. The thesis follows since Lemma 2 implies that $\left|\xi_{M I N}\right| \geq \frac{|\phi|}{2}$.

Observe that in the best case $\nu_{i j}=|\phi|$, thus the bound becomes

$$
\frac{\left|\xi_{i j}^{(\min )}\right|}{\left|\xi_{M I N}\right|} \leq \frac{\left|\xi_{i j}\right|}{\left|\xi_{M I N}\right|} \leq 2
$$

while in the worst case $\nu_{i j}=1$ and we have

$$
\frac{\left|\xi_{i j}^{(\min )}\right|}{\left|\xi_{M I N}\right|} \leq \frac{\left|\xi_{i j}\right|}{\left|\xi_{M I N}\right|} \leq 4-\frac{2}{|\phi|} \leq 4 .
$$

Theorem 3 shows a similar result for the EP-SOPs with remainder.

Theorem 3. Let $\psi_{M I N}$ be a minimal EP-SOP with remainder of a Boolean function $f$, and $\phi$ be a minimal SOP form for $f$.

Let $\psi_{i j}^{(\text {min })}$ be the minimal $(i, j)$-EP-SOP with remainder derived with respect to the couple of variables $x_{i}$ and $x_{j}$ appearing with the highest frequency in the products of $\phi$. Then

$$
\frac{\left|\psi_{i j}^{(\min )}\right|}{\left|\psi_{M I N}\right|} \leq \frac{\left|\psi_{i j}\right|}{\left|\psi_{M I N}\right|} \leq 2 .
$$

Proof. First observe that

$$
\left|\psi_{i j}^{(\min )}\right| \leq\left|\psi_{i j}\right|=|\phi|
$$


since each product of $\phi$ appears only once in $\psi_{i j}$, in one of the two factors $\phi_{\oplus}^{\prime}$ and $\phi_{\bar{\oplus}}^{\prime}$, or in the remainder $\rho$, and the two expressions $\phi_{\oplus}^{\prime}$ and $\phi_{\bar{\oplus}}^{\prime}$ are further minimized. Moreover, Lemma 3 implies that

$$
\left|\psi_{M I N}\right| \geq \frac{1}{2}(|\phi|+|\rho|) \text {. }
$$

Now suppose that the projections in $\psi_{M I N}$ are performed with respect to the variables $x_{h}$ and $x_{k}$. Thus, since $|\rho|=|\phi|-\nu_{h k}$, with $\nu_{h k} \leq \nu_{i j}$ and $\nu_{i j} \leq|\phi|$, we get

$$
\frac{\left|\psi_{i j}^{(\min )}\right|}{\left|\psi_{M I N}\right|} \leq \frac{\left|\psi_{i j}\right|}{\left|\psi_{M I N}\right|} \leq \frac{|\phi|}{|\phi|-\nu_{i j} / 2} \leq \frac{|\phi|}{|\phi|-|\phi| / 2} \leq 2 .
$$

Note that Theorem 2 and Theorem 3 show that the approximation ratios hold even if the factors $\phi_{\oplus}, \phi_{\bar{\oplus}}, \phi_{\oplus}^{\prime}$ and $\phi_{\bar{\oplus}}^{\prime}$ are not minimized. Therefore, the algorithms proposed are indeed polynomial approximation algorithms for the given problems. The resulting EP-SOP without remainder $\xi_{i j}$ has a size that can be upper bounded by $\left(4-2 \nu_{i j} /|\phi|\right)\left|\xi_{M I N}\right|$, i.e., in the worst case by $4\left|\xi_{M I N}\right|$, while the EP-SOP with remainder $\psi_{i j}$ has a size that can be upper bounded by $2\left|\psi_{M I N}\right|$.

As a final observation, we would like to point out that the couple of variables, say $x_{i}$ and $x_{j}$, with the highest frequency in general does not guarantee that $\xi_{M I N}=\xi_{i j}^{(\min )}$ and $\psi_{M I N}=\psi_{i j}^{(\min )}$, as the following counterexample shows.

Example 2. Let us consider the minimal SOP $\phi=x_{1} x_{2} x_{3} x_{4}+x_{1} x_{2} \bar{x}_{3} \bar{x}_{4}+\bar{x}_{1} x_{2}$. We want to find the two minimal EP-SOP forms. The couple of variables with the highest frequency is $x_{1}$ and $x_{2}$.

The approximation algorithm computes the following form without remainder:

$$
\xi_{12}^{(\min )}=\left(x_{1} \oplus x_{2}\right) x_{2}+\left(x_{1} \oplus \bar{x}_{2}\right)\left(x_{2} x_{3} x_{4}+x_{2} \bar{x}_{3} \bar{x}_{4}\right)
$$

and the following form with remainder:

$$
\psi_{12}^{(\min )}=\left(x_{1} \oplus x_{2}\right) x_{2}+\left(x_{1} \oplus \bar{x}_{2}\right)\left(x_{2} x_{3} x_{4}+x_{2} \bar{x}_{3} \bar{x}_{4}\right),
$$

while the minimal solutions are $\xi_{M I N}=\xi_{34}^{(\min )}=\left(x_{3} \oplus \bar{x}_{4}\right) x_{2}+\left(x_{3} \oplus x_{4}\right)\left(\bar{x}_{1} x_{2}\right)$ and $\psi_{M I N}=\psi_{34}^{(\min )}=\left(x_{3} \oplus \bar{x}_{4}\right)\left(x_{1} x_{2}\right)+\bar{x}_{1} x_{2}$, respectively.

\section{Experimental results}

In this section we discuss the computational results obtained by applying the polynomial approximation algorithm presented above to the standard ESPRESSO benchmark suite [20]. We consider four different variants of our algorithm. In fact, we address the minimization of EP-SOP forms both with and without remainder, in order to estimate the practical utility of either form. Moreover, as 
most benchmarks have multiple outputs, the definition of the most frequent couple of variables can be referred either to the whole set of outputs (global frequency) or to each single output (local frequency). In the former case, we will determine a single EP-SOP form, projecting the original minimal SOP form $\phi$ with respect to the couple of variables appearing in the largest number of products of $\phi$. In the latter case, we will find the most frequent couple of variables for each different output and perform independent projections, obtaining separate EP-SOP forms for the outputs which have been projected onto different couples of subspaces. In both cases all the SOP forms are synthesized together with multi-output synthesis. Combining the two approaches related to the use of the remainder and the two approaches related to the global and local frequency, we obtain four different algorithms, respectively denoted as NG (no remainder and global frequency), NL (no remainder and local frequency), RG (remainder and global frequency), RL (remainder and local frequency).

All computational experiments were performed on a Pentium $1.6 \mathrm{GHz}$ processor with 1 GB RAM. We report in the following a significant subset of the experiments.

Table 1 reports a cost-oriented comparison among the original optimal SOP form determined by ESPRESSO EXACT and the EP-SOP forms yielded by the four algorithms: the first column reports the name of the instance, the following five triples of columns report the computational time in seconds, together with the area and the delay of physical implementations for the five expressions. These were evaluated using a technology mapping (mcnc.genlib) provided by the SIS [18] tool.

The computational time for the EP-SOP forms does not include the time required to compute the optimal SOP form (which is shown in the second column), but only the time to factorize it and to heuristically minimize its projections. As the results show, the overhead added by the last two steps is quite limited.

Of course, the physical implementation of the EP-SOP forms also includes one or more EXOR gates, whose cost cannot be neglected, as our results clearly show. First of all, the EXOR part of the network can be expensive, depending on the technology adopted. Second, some functions benefit from the multi-output minimization: common products can be shared, thus reducing the overall area. Comparing the performances of the four algorithms one to another, we can note how this fact particularly affects the performance of the algorithms NL and RL referring to the local definition of frequency, while the algorithm performing better seems to be the RG algorithm.

It should be noticed, however, that the gain obtained by the EP-SOP form is on about the $35 \%$ of instances, and can be quite striking: the gain on instance adr4 exceeds $50 \%$ and for many other instances (e.g., root, z4) it exceeds $40 \%$.

Apart from algorithm NL, which only equals some best result, never hitting one alone, even the less effective of the other three algorithms, that is RL, improves by $45 \%$ the cost of instance $f 51 \mathrm{~m}$.

Given that the time required to obtain such improvements is rather limited, evaluating the EP-SOP forms as a possible alternative to the optimal SOP form 
appears to be an advisable post-processing strategy. We have further investigated

\begin{tabular}{|c|c|c|c|c|c|c|c|c|c|c|c|c|c|c|c|}
\hline \multirow[b]{2}{*}{ Bench. } & \multirow{2}{*}{\multicolumn{3}{|c|}{$\begin{array}{c}\text { min SOP } \\
\text { CPU area delay }\end{array}$}} & \multicolumn{12}{|c|}{ min EP-SOP } \\
\hline & & & & \multicolumn{3}{|c|}{\begin{tabular}{|c|} 
NG \\
CPU area delay \\
\end{tabular}} & \multicolumn{3}{|c|}{\begin{tabular}{|c|} 
NL \\
CPU area delay
\end{tabular}} & \multicolumn{3}{|c|}{\begin{tabular}{|c|} 
RG \\
CPU area delay
\end{tabular}} & \multicolumn{3}{|c|}{$\begin{array}{c}\text { RL } \\
\text { CPU area delay }\end{array}$} \\
\hline $\operatorname{addm} 4$ & 0.14 & 1172 & 47.9 & 0.06 & 1291 & 52.5 & 0.06 & 975 & 40.4 & 0.04 & 1101 & 48.5 & 0.07 & 906 & 38.3 \\
\hline adr4 & 0.04 & 224 & 19.2 & 0.03 & 174 & 15.2 & 0.03 & 155 & 16.0 & 0.03 & 105 & 11.1 & 0.04 & 141 & 13.5 \\
\hline md & 0.06 & 1171 & 46.7 & 0.03 & 1082 & 43.5 & 0.05 & 1040 & 39.1 & 0.03 & 1046 & 42.4 & 0.06 & 1022 & 38.0 \\
\hline b2 & 0.23 & 3876 & 79.8 & 0.06 & 4113 & 81.3 & 0.06 & 4180 & 81.3 & 0.04 & 4169 & 82.6 & 0.04 & 4242 & 82.6 \\
\hline b4 & 3.45 & 645 & 30.5 & 0.01 & 802 & 33.3 & 0.01 & 841 & 33.1 & 0.01 & 717 & 34.4 & 0.01 & 779 & 32.8 \\
\hline br1 & 0.01 & 446 & 32.5 & 0.02 & 353 & 24.5 & 0.02 & 381 & 25.7 & 0.02 & 353 & 24.5 & 0.02 & 381 & 25.7 \\
\hline br2 & 0.01 & 352 & 26.6 & 0.01 & 292 & 25.5 & 0.01 & 314 & 30.0 & 0.01 & 292 & 25.5 & 0.01 & 314 & 30.0 \\
\hline chkn & 0.48 & 717 & 43.6 & 0.04 & 832 & 42.2 & 0.06 & 777 & 39.2 & 0.01 & 758 & 36.1 & 0.01 & 764 & 46.7 \\
\hline dc2 & 0.04 & 253 & 23.1 & 0.01 & 286 & 22.4 & 0.01 & 236 & 19.7 & 0.01 & 263 & 21.7 & 0.01 & 236 & 19.7 \\
\hline exps & 0.50 & 3932 & 114.5 & 0.06 & 3778 & 114.8 & 0.06 & 3900 & 104.6 & 0.08 & 3760 & 112.6 & 0.09 & 3877 & 106.4 \\
\hline $\mathrm{f} 51 \mathrm{~m}$ & 0.09 & 501 & 31.5 & 0.04 & 413 & 26.2 & 0.04 & 339 & 26.4 & 0.04 & 311 & 20.5 & 0.04 & 273 & 19.1 \\
\hline ino & 0.10 & 1214 & 48.3 & 0.03 & 1056 & 48.1 & 0.05 & 1015 & 42.5 & 0.05 & 1019 & 48.0 & 0.06 & 989 & 44.9 \\
\hline in1 & 0.23 & 3876 & 79.8 & 0.06 & 4113 & 81.3 & 0.06 & 4180 & 81.3 & 0.06 & 4169 & 82.6 & 0.06 & 4242 & 82.6 \\
\hline in2 & 0.09 & 1112 & 41.4 & 0.03 & 1000 & 36.7 & 0.01 & 1041 & 37.3 & 0.03 & 1002 & 37.3 & 0.03 & 1039 & 37.9 \\
\hline in5 & 0.14 & 905 & 38.5 & 0.01 & 976 & 39.2 & 0.01 & 1040 & 37.2 & 0.01 & 923 & 40.9 & 0.01 & 993 & 39.7 \\
\hline intb & 2.96 & 2170 & 57.3 & 0.44 & 3392 & 75.5 & 0.83 & 2693 & 63.2 & 0.34 & 2466 & 57.6 & 0.67 & 2526 & 61.6 \\
\hline luc & 0.01 & 806 & 41.0 & 0.01 & 779 & 52.8 & 0.01 & 883 & 51.8 & 0.01 & 758 & 52.4 & 0.01 & 862 & 50.6 \\
\hline m1 & 0.01 & 208 & 19.6 & .03 & 304 & 21.0 & 0.03 & 352 & 21.2 & 0.03 & 308 & 22.8 & 0.03 & 356 & 22.8 \\
\hline m2 & 0.01 & 710 & 37.8 & 0.01 & 833 & 40.9 & 0.01 & 893 & 40.5 & 0.01 & 861 & 42.5 & 0.01 & 921 & 41.9 \\
\hline m3 & 0.04 & 839 & 38.3 & 0.01 & 1286 & 48.4 & 0.01 & 1283 & 52.2 & 0.01 & 1172 & 51.7 & 0.01 & 1235 & 54.4 \\
\hline m181 & 0.60 & 166 & 18.4 & 0.01 & 327 & 22.4 & 0.03 & 311 & 24.9 & 0.01 & 240 & 22.5 & 0.01 & 267 & 19.8 \\
\hline $\max 128$ & 0.09 & 1292 & 58.0 & 0.09 & 2055 & 71.6 & 0.09 & 2194 & 77.6 & 0.07 & 2098 & 71.5 & 0.07 & 1975 & 72.4 \\
\hline mlp4 & 0.31 & 734 & 36.4 & 0.03 & 983 & 43.0 & 0.04 & 891 & 40.1 & 0.03 & 839 & 40.5 & 0.03 & 857 & 40.1 \\
\hline mp2d & 0.25 & 362 & 26.0 & 0.01 & 428 & 25.3 & 0.01 & 420 & 28.9 & 0.01 & 333 & 23.7 & 0.01 & 360 & 25.5 \\
\hline newcond & 0.01 & 114 & 17.4 & 01 & 132 & 18.6 & 0.01 & 124 & 18.6 & 0.01 & 119 & 18.2 & 0.01 & 124 & 18.6 \\
\hline p82 & 0.01 & 239 & 18.4 & 0.01 & 239 & 25.8 & 0.01 & 302 & 23.9 & 0.01 & 241 & 25.0 & 0.01 & 309 & 24.7 \\
\hline radd & 0.39 & 183 & 15.7 & 0.01 & 196 & 18.9 & 0.01 & 181 & 19.5 & 0.01 & 120 & 15.1 & 0.01 & 158 & 16.8 \\
\hline rckl & 0.04 & 341 & 49.7 & 0.01 & 495 & 72.3 & 0.01 & 519 & 72.3 & 0.01 & 495 & 72.3 & 0.01 & 519 & 72.3 \\
\hline $\mathrm{rd73}$ & 0.03 & 220 & 25.6 & 0.03 & 389 & 27.6 & 0.03 & 308 & 28.4 & 0.03 & 339 & 26.9 & 0.03 & 264 & 24.1 \\
\hline risc & 0.01 & 228 & 18.7 & 0.02 & 312 & 29.0 & 0.02 & 435 & 32.7 & 0.03 & 310 & 29.0 & 0.02 & 434 & 32.5 \\
\hline root & 0.35 & 592 & 35.5 & 0.02 & 367 & 27.7 & 0.02 & 380 & 25.3 & 0.03 & 349 & 26.5 & 0.03 & 350 & 25.7 \\
\hline sqr6 & 0.06 & 278 & 25.5 & 0.01 & 397 & 27.0 & 0.01 & 462 & 26.2 & 0.01 & 330 & 24.9 & 0.01 & 405 & 26.2 \\
\hline t3 & 0.40 & 186 & 21.5 & 0.02 & 193 & 16.2 & 0.03 & 213 & 15.8 & 0.02 & 180 & 19.8 & 0.02 & 206 & 19.7 \\
\hline tms & 0.03 & 587 & 35.4 & 0.01 & 675 & 35.2 & 0.01 & 754 & 35.5 & 0.01 & 675 & 35.2 & 0.01 & 754 & 35.5 \\
\hline vg2 & 0.53 & 341 & 18.6 & 0.04 & 628 & 25.7 & 0.06 & 581 & 26.0 & 0.03 & 468 & 22.5 & 0.04 & 500 & 21.4 \\
\hline$v \operatorname{tx1}$ & 0.17 & 324 & 21.3 & 0.01 & 441 & 25.5 & 0.01 & 497 & 21.1 & 0.01 & 365 & 23.4 & 0.01 & 465 & 20.7 \\
\hline$x 6 d n$ & 0.18 & 1054 & 36.8 & 0.01 & 854 & 34.9 & 0.01 & 870 & 34.9 & 0.01 & 817 & 34.8 & 0.01 & 834 & 34.8 \\
\hline$x 9 d n$ & 0.20 & 384 & 23.0 & 0.04 & 496 & 25.4 & 0.06 & 560 & 24.2 & 0.04 & 424 & 24.7 & 0.03 & 528 & 22.6 \\
\hline $\mathrm{z} 4$ & 0.01 & 171 & 18.3 & 0.01 & 159 & 18.6 & 0.01 & 165 & 20.6 & 0.01 & 99 & 14.2 & 0.01 & 132 & 17.9 \\
\hline
\end{tabular}

Table 1. Synthesis time, area and delay of EP-SOP and SOP forms (computed in SIS after the technology mapping).

whether the Boolean factorization proposed in the present paper actually differs from similar techniques already known in the literature and applied in synthesis tools. We have applied the multilevel synthesis routines (script.rugged) of SIS to the optimal SOP forms and to the four EP-SOP forms, in order to find out whether they end up with a similar final structure or not. The first remark that can be done is that in some cases (e.g., b2, exps and in1), SIS was unable to process the optimal SOP form (in a limit time of 12 hours). Starting from the EP-SOP forms, this happened only for instance in1, and only for the two EPSOP forms with remainder. Only few times the final results were identical (10\%), and half of the times the final result obtained starting from an EP-SOP form 
was better than the one obtained from the optimal SOP form, ranging from $30 \%$ better to $30 \%$ worse.

\section{Conclusion}

Although deriving an optimal EP-SOP form from an optimal SOP form is an $N P^{N P}$-hard problem, in this paper we have described a polynomial time approximation algorithm which guarantees a near-optimal solution. We propose this algorithm as a post-processing step after the SOP synthesis, in order to possibly reduce the area of the resulting networks. Our experiments show that in about $35 \%$ of the considered benchmarks the area obtained is smaller, sometimes even by $40-50 \%$.

It could be an interesting development to study different kinds of projection, such as dividing the Boolean space into subspaces whose characteristic functions are represented by EXORs with more than two literals. Given the similar nature of the problem, it could also be interesting to study the relationship between Linear Transformed BDDs [10] and EP-SOP forms.

\section{References}

1. A. Bernasconi, V. Ciriani, F. Luccio, and L. Pagli. Three-Level Logic Minimization Based on Function Regularities. IEEE Transactions on TCAD, 22(8):1005-1016, 2003.

2. R. Brayton, G. Hachtel, C. McMullen, and A. Sangiovanni-Vincentelli. Logic Minimization Algorithms for VLSI Synthesis. Kluwer Ac. Pub., 1984.

3. G. Caruso. Near Optimal Factorization of Boolean Functions. IEEE Transactions on $C A D, 10(8): 1072-1078,1991$.

4. O. Coudert. Two-Level Logic Minimization: an Overview. INTEGRATION, 17:97140, 1994.

5. D. Debnath and T. Sasao. A Heuristic Algorithm to Design AND-OR-EXOR Three-Level Networks. In Asia and South Pacific Design Automation Conference, pages 69-74, 1998.

6. D. Debnath and Z. Vranesic. A Fast Algorithm for OR-AND-OR Synthesis. IEEE Transactions on CAD, 22(9):1166-1176, 2003.

7. E. Dubrova, D. Miller, and J. Muzio. AOXMIN-MV: A Heuristic Algorithm for AND-OR-XOR Minimization. In 4th Int. Workshop on the Applications of the Reed Muller Expansion in circuit Design, pages 37-54, 1999.

8. M. Fujita, Y. Matsunaga, and M. Ciesielski. Multi-Level Logic Optimization. In S. Hassoun and T. Sasao, editors, Logic Synthesis and Verification, pages 29-63. Kluwer Academic Publishers, 2002.

9. M. Garey and D. Johnson. Computer and Intractability: A Guide to the Theory of NP-completeness. W.H. Freeman and Company, 1979.

10. W. Günther and R. Drechsler. On the Computational Power of LinearlyTransformed BDDs. Information Processing Letters, 75(3):119-125, 2000.

11. W. Günther and R. Drechsler. Efficient Minimization and Manipulation of Linearly-Transformed Binary Decision Diagrams. IEEE Transaction on Computers, 52(9):1196-1209, 2003. 
12. F. Luccio and L. Pagli. On a New Boolean Function with Applications. IEEE Transactions on Computers, 48(3):296-310, 1999.

13. P. McGeer, J. Sanghavi, R. Brayton, and A. Sangiovanni-Vincentelli. EspressoSignature: A New Exact Minimizer for Logic Functions. IEEE Transactions on VLSI, 1(4):432-440, 1993.

14. M. Perkowski. A New Representation of Strongly Unspecified Switching Functions and its Application to Multi-Level AND/OR/EXOR Synthesis. In IFIP WG 10.5 Workshop on Applications of the Reed-Muller Expansion, pages 143-151, 1995.

15. T. Sasao. On the Complexity of Three-Level Logic Circuits. In Int. Workshop on Logic Synthesis, 1989.

16. T. Sasao. A Design Method for AND-OR-EXOR Three Level Networks. In Int. Workshop on Logic Synthesis, pages 8:11-8:20, 1995.

17. T. Sasao. Switching Theory for Logic Synthesis. Kluwer Academic Publishers, 1999.

18. E. Sentovich, K. Singh, L. Lavagno, C. Moon, A. S. R. Murgai, H. Savoj, P. Stephan, R. Brayton, and A. Sangiovanni-Vincentelli. SIS: A system for sequential circuit synthesis. Technical report, 1992.

19. C. Umans, T. Villa, and A. Sangiovanni-Vincentelli. Complexity of Two-Level Logic Minimization. IEEE Transactions on Computer-Aided Design, to appear.

20. S. Yang. Logic synthesis and optimization benchmarks user guide version 3.0. User guide, Microelectronic Center, 1991. 